\title{
Amin Hosseini \\ What can be expected from a cubic derivation on finite dimensional algebras?
}

Received: 28 September 2016 / Accepted: 2 April 2017 / Published online: 17 April 2017

(C) The Author(s) 2017. This article is an open access publication

\begin{abstract}
In this paper, we prove that every rank one cubic derivation on a unital integral domain is identically zero. From this conclusion, under certain conditions, we achieve that the image of a cubic derivation on a commutative algebra is contained in the Jacobson radical of algebra. As the main result of the current study, we prove that every cubic derivation on a finite dimensional algebra, under some circumstances, is identically zero.
\end{abstract}

Mathematics Subject Classification $\quad 47 \mathrm{~B} 47 \cdot 47 \mathrm{~B} 48$

في هذه الورقة، نثبت أن أي اشتقاق تكعيبي من الرتبة الأولى على مجال صحيح واحدي يطابق الصفر . من هذا الاستنتاج، وفي ظل شروط معينة، نثبت

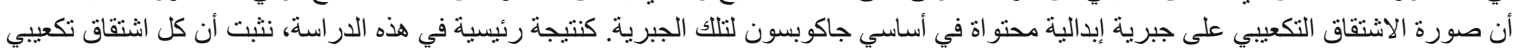

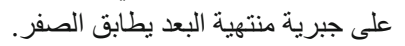

\section{Introduction and preliminaries}

Throughout this paper, $\mathcal{A}$ denotes an associative complex algebra. If $\mathcal{A}$ is unital, then $\mathbf{1}$ stands for its unit element. Before describing the aim of the paper, let us recall some basic definitions and set the notations which we use in the sequel. An algebra $\mathcal{A}$ is said to be a domain if $\mathcal{A} \neq\{0\}$, and either $a=0$ or $b=0$, whenever $a b=0$ in $\mathcal{A}$. A commutative algebra which is also a domain is called an integral domain. As usual, $\operatorname{rad}(\mathcal{A})$ denotes the Jacobson radical of $\mathcal{A}$. Recall that the Jacobson radical is the intersection of all primitive ideals of an algebra. An algebra $\mathcal{A}$ is called semi-simple if $\operatorname{rad}(\mathcal{A})=\{0\}$. A non-zero linear functional $\varphi$ is called a character if $\varphi(a b)=\varphi(a) \varphi(b)$ for every $a, b \in \mathcal{A}$. By $\Phi_{\mathcal{A}}$, we denote the set of all characters on $\mathcal{A}$. We know that $\operatorname{ker} \varphi$ is a maximal ideal of $\mathcal{A}$ for each $\varphi \in \Phi_{\mathcal{A}}$ (see [5]). Recall that a linear mapping $d: \mathcal{A} \rightarrow \mathcal{A}$ is called a derivation if it satisfies the Leibnitz's rule $d(a b)=d(a) b+a d(b)$ for all $a, b \in \mathcal{A}$. In [7], Eshaghi Gordji et al. introduced the concept of a cubic derivation. A mapping $d: \mathcal{A} \rightarrow \mathcal{A}$ is called a cubic derivation if $d$ is a cubic homogeneous mapping, that is $d(\lambda a)=\lambda^{3} d(a)(\lambda \in \mathbb{C}, a \in \mathcal{A})$, and $d(a b)=d(a) b^{3}+a^{3} d(b)$ for all $a, b \in \mathcal{A}$. The research to date has been focused on investigating stability of cubic derivations (see $[7,8,12,26])$. In the present note, we are going to investigate this concept from another perspective. The image of derivations has a fairly long history. Results on the image of derivations defined on Banach algebras comprise a fruitful area of research intensively developed during the last sixty years (see [9,13-15,17,24,25]). After considerations regarding this topic, no article was found to study the image of cubic derivations. In this paper, we establish some permanence results of cubic derivations. Here, we are planning to prove some results similar to Singer-Wermer theorem and Thomas theorem for cubic derivations. In 1955, Singer and Wermer [24] achieved

A. Hosseini $(\varangle)$

Department of Mathematics, Kashmar Higher Education Institute, Kashmar, Iran

E-mail: hosseini.amin82@gmail.com 
a fundamental result which started investigation into the range of derivations on Banach algebras. The so-called Singer-Wermer theorem states that any continuous derivation on a commutative Banach algebra maps into the Jacobson radical. In the same paper, they made a very insightful conjecture that the assumption of continuity is superfluous. In 1988, Thomas [25] proved the conjecture. According to this result, every derivation on a commutative Banach algebra maps the algebra into its radical. To achieve some results on the image of cubic derivations, we prove that every rank one cubic derivation on a unital integral domain is identically zero. As a conclusion from this, it is proved that if $\mathcal{A}$ is a unital algebra and $d: \mathcal{A} \rightarrow \mathcal{A}$ is a cubic derivation such that $d(a)-d(b) \in \operatorname{ker} \varphi$ whenever $a-b \in \operatorname{ker} \varphi$ for every $a, b, \in \mathcal{A}$ and $\varphi \in \Phi_{\mathcal{A}}$, then $d(\mathcal{A}) \subseteq \bigcap_{\varphi \in \Phi_{\mathcal{A}}} \operatorname{ker} \varphi$. In particular, if $\mathcal{A}$ is also commutative, then $d(\mathcal{A}) \subseteq \operatorname{rad}(\mathcal{A})$. At the end of the paper, we are going to make clear the status of cubic derivations on some finite dimensional algebras. To reach this goal, let $n$ be a positive integer and $\mathcal{A}$ be an $n$-dimensional unital algebra with the basis $\mathcal{B}=\left\{b_{1}, b_{2}, \ldots, b_{n}\right\}$. Furthermore, suppose that for every integer $k, 1 \leq k \leq n$, an ideal $\mathcal{J}_{k}$ generated by $\mathcal{B}-\left\{b_{k}\right\}$, is a proper subset of $\mathcal{A}$. If $d: \mathcal{A} \rightarrow \mathcal{A}$ is a cubic derivation such that $d(a)-d(b) \in \mathcal{J}_{\kappa}$ whenever $a-b \in \mathcal{J}_{\kappa}$ for every $a, b \in \mathcal{A}$ and $1 \leq k \leq n$, then $d$ is identically zero.

\section{Results and proofs}

Throughout the following discussion, an operation $T: \mathcal{A} \rightarrow \mathcal{A}$ is called a rank one operator if there exist a non-zero element $x$ of $\mathcal{A}$ and a functional $\psi: \mathcal{A} \rightarrow \mathbb{C}$ such that $T(a)=\psi(a) x$ for all $z \in \mathcal{A}$. Since the case $\Psi_{\mathcal{A}}=\phi$ makes everything trivial, we consider $\Phi_{\mathcal{A}}$ ad a non-empty set. Now, we begin with the following theorem.

\section{Theorem 2.1 Every rank one cubic derivation on a unital integral domain is identically zero.}

Proof Let $\mathcal{A}$ be a unital integral domain. To obtain a contradiction, suppose that there exists a non-zero element $a_{0} \in \mathcal{A}$ such that $d\left(a_{0}\right) \neq 0$. Since $d$ is a rank one cubic derivation, there exist a non-zero element $x$ of $\mathcal{A}$ and a functional $\psi: \mathcal{A} \rightarrow \mathbb{C}$ such that $d(a)=\psi(a) x$ for all $a \in \mathcal{A}$. Evidently, $\psi\left(a_{0}\right) \neq 0$, and $d(\mathbf{1})=0$. Assume that $d(x)=0$. So, $\psi(x) x=0$ and it implies that $\psi(x)=0$. We have $\psi\left(a_{0}^{2}\right) x=d\left(a_{0}^{2}\right)=d\left(a_{0}\right) a_{0}^{3}+a_{0}^{3} d\left(a_{0}\right)=$ $2 a_{0}^{3} d\left(a_{0}\right)=2 a_{0}^{3} \psi\left(a_{0}\right) x=2 \psi\left(a_{0}\right) a_{0}^{3} x$. Therefore, we have

$$
\begin{aligned}
0 & =\left(\psi\left(a_{0}^{2}\right)\right)^{3} d(x)=d\left(\psi\left(a_{0}^{2}\right) x\right)=d\left(2 \psi\left(a_{0}\right) a_{0}^{3} x\right) \\
& =8\left(\psi\left(a_{0}\right)\right)^{3}\left[d\left(a_{0}^{3}\right) x^{3}+a_{0}^{9} d(x)\right] \\
& =8\left(\psi\left(a_{0}\right)\right)^{3} d\left(a_{0}^{3}\right) x^{3}
\end{aligned}
$$

Previous equation along with the assumption that $\mathcal{A}$ is a domain implies that $d\left(a_{0}^{3}\right)=0$. Thus,

$$
\begin{aligned}
0 & =d\left(a_{0}^{3}\right)=d\left(a_{0}^{2} a_{0}\right)=d\left(a_{0}^{2}\right) a_{0}^{3}+a_{0}^{6} d\left(a_{0}\right) \\
& =\left[d\left(a_{0}\right) a_{0}^{3}+a_{0}^{3} d\left(a_{0}\right)\right] a_{0}^{3}+a_{0}^{6} d\left(a_{0}\right) \\
& =3 a_{0}^{6} d\left(a_{0}\right) .
\end{aligned}
$$

Reusing the hypothesis that $\mathcal{A}$ is a domain, we obtain that $d\left(a_{0}\right)=0$ which it is a contradiction. Now suppose that $d(x) \neq 0$. This implies that $\psi(x) \neq 0$. Note that

$$
\psi\left(x^{2}\right) x=d\left(x^{2}\right)=2 x^{3} d(x)=2 \psi(x) x^{4} .
$$

If $\psi\left(x^{2}\right)=0$, then it follows from Eq. (2.1) that either $\psi(x)=0$ or $x^{4}=0$, but we know that both of them are non-zero. This contradiction shows that $\psi\left(x^{2}\right)$ must be non-zero. Putting $\frac{\psi\left(x^{2}\right)}{2 \psi(x)}=h$ in Eq. (2.1), we have $x^{4}-h x=0$; factoring out $x$ on the left, we obtain that $x\left(x^{3}-h \mathbf{1}\right)=0$. Consequently, either $x=0$ which it is a contradiction, or $x^{3}=h \mathbf{1}$. We, therefore, have $0=h^{3} d(\mathbf{1})=d(h \mathbf{1})=d\left(x^{3}\right)=d\left(x x^{2}\right)=$ $d(x) x^{6}+x^{3} d\left(x^{2}\right)=3 x^{6} d(x)$. Since $\mathcal{A}$ is a domain, either $x^{6}=0$ or $d(x)=0$, which it is a contradiction. This contradiction shows that there is no element $a_{0}$ of $\mathcal{A}$ such that $d\left(a_{0}\right) \neq 0$. Therefore, $d$ is identically zero.

Now, we present the following corollary. 
Corollary 2.2 Every rank one derivation on a unital integral domain is identically zero.

Proof See the above proof.

In the following corollary, we achieve a result similar to Thomas's theorem for the image of cubic derivations.

Corollary 2.3 Let $\mathcal{A}$ be a unital algebra and $d: \mathcal{A} \rightarrow \mathcal{A}$ be a cubic derivation. If $d(a)-d(b) \in$ ker $\varphi$ whenever $a-b \in \operatorname{ker} \varphi$ for every $a, b \in \mathcal{A}$ and $\varphi \in \Phi_{\mathcal{A}}$, then $d(\mathcal{A}) \subseteq \bigcap_{\varphi \in \Phi_{\mathcal{A}}} \operatorname{ker} \varphi$. In particular, if $\mathcal{A}$ is also commutative, then $d(\mathcal{A}) \subseteq \operatorname{rad}(\mathcal{A})$.

Proof For an arbitrary element $\varphi \in \Phi_{\mathcal{A}}$, define a map $D: \frac{\mathcal{A}}{\operatorname{ker} \varphi} \rightarrow \frac{\mathcal{A}}{\operatorname{ker} \varphi}$ by $D(a+\operatorname{ker} \varphi)=d(a)+\operatorname{ker} \varphi$ for every $a \in \mathcal{A}$. Evidently, the algebra $\frac{\mathcal{A}}{\operatorname{ker} \varphi}$ is a unital, integral domain (i.e., it is a unital, commutative domain) and it follows from Proposition 1.3.37 of [5] that $\operatorname{dim}\left(\frac{\mathcal{A}}{\operatorname{ker} \varphi}\right)=1$. So, $D$ is a rank one cubic derivation on the unital integral domain $\frac{\mathcal{A}}{\operatorname{ker} \varphi}$. Now, Theorem 2.1 implies that the cubic derivation $D$ is identically zero. It means that $d(\mathcal{A}) \subseteq \operatorname{ker} \varphi$ and since we are assuming $\varphi$ is an arbitrary element of $\Phi_{\mathcal{A}}, d(\mathcal{A}) \subseteq \bigcap_{\varphi \in \Phi_{\mathcal{A}}} \operatorname{ker} \varphi$. It is obvious that if $\mathcal{A}$ is commutative, then $\bigcap_{\varphi \in \Phi_{\mathcal{A}}} \operatorname{ker} \varphi=\operatorname{rad}(\mathcal{A})$ (see [5]). Thus, the last assertion is also proved.

At the end, we observe cubic derivations on unital finite-dimensional algebras. So, let $n$ be a positive integer and $\mathcal{A}$ be an $n$-dimensional unital algebra with the basis $\mathcal{B}=\left\{b_{1}, b_{2}, \ldots, b_{n}\right\}$. We prove the following theorem.

Theorem 2.4 Suppose that for every integer $k, 1 \leq k \leq n$, an ideal $\mathcal{J}_{k}$ generated by $\mathcal{B}-\left\{b_{k}\right\}$ is a proper subset of $\mathcal{A}$. If $d: \mathcal{A} \rightarrow \mathcal{A}$ is a cubic derivation such that $d(a)-d(b) \in \mathcal{J}_{k}$ whenever $a-b \in \mathcal{J}_{k}$ for every $a, b \in \mathcal{A}, 1 \leq k \leq n$, then $d$ is identically zero.

Proof Evidently, $\operatorname{dim}\left(\frac{\mathcal{A}}{\mathcal{J}_{k}}\right)=1$ for every $k \in\{1,2, \ldots, n\}$. We claim that $\mathcal{J}_{k}$ is a maximal ideal of $\mathcal{A}$ for each $k \in\{1,2, \ldots, n\}$. Namely, if $\mathcal{J}_{k}$ is not a maximal ideal of $\mathcal{A}$ for some $k, 1 \leq k \leq n$, then there exists a maximal ideal $\mathcal{M}_{k}$ of $\mathcal{A}$ such that $\mathcal{J}_{k} \subset \mathcal{M}_{k} \subset \mathcal{A}$. But then $n-1=\operatorname{dim}\left(\mathcal{J}_{k}\right)<\operatorname{dim}\left(\mathcal{M}_{k}\right)<n$, a contradiction. Hence, every $\mathcal{J}_{k}$ must be a maximal ideal of $\mathcal{A}$. It follows from Corollary 1.3 .45 of [5] that every $\mathcal{J}_{k}$ is a prime ideal. Moreover, according to Proposition 1.3.37 of [5] for every maximal ideal $\mathcal{J}_{k}(1 \leq k \leq n)$, there exists a character $\varphi_{k} \in \Phi_{\mathcal{A}}$ such $\mathcal{J}_{k}=\operatorname{ker} \varphi_{k}$. So, the algebra $\frac{\mathcal{A}}{\mathcal{J}_{k}}=\frac{\mathcal{A}}{\operatorname{ker} \varphi_{k}}$ is an integral domain. Based on the aforementioned assumption, we have $d(a)-d(b) \in \mathcal{J}_{k}$ whenever $a-b \in \mathcal{J}_{k}$ for every $a, b \in \mathcal{A}$ and $1 \leq k \leq n$. Therefore, it follows from Theorem 2.1 that the cubic derivation $D: \frac{\mathcal{A}}{\mathcal{J}_{k}} \rightarrow \frac{\mathcal{A}}{\mathcal{J}_{k}}$ defined by $D\left(a+\mathcal{J}_{k}\right)=d(a)+\mathcal{J}_{k}$ is identically zero. It means that $d(\mathcal{A}) \subseteq \mathcal{J}_{k}$, for every $k \in\{1,2, \ldots, n\}$. Hence, $d(\mathcal{A}) \subseteq \bigcap_{k=1}^{n} \mathcal{J}_{k}$. Assume that there is an element $a_{0}$ of $\mathcal{A}$ such that $d\left(a_{0}\right) \neq 0$. Since $\mathcal{B}=\left\{b_{1}, b_{2}, \ldots, b_{n}\right\}$ is a basis for $\mathcal{A}$, there exist the complex numbers $\alpha_{i_{j}}$, and the elements $b_{i_{j}}$ of $\mathcal{B}$ such that

$$
d\left(a_{0}\right)=\sum_{j=1}^{m} \alpha_{i_{j}} b_{i_{j}}=\alpha_{i_{1}} b_{i_{1}}+\alpha_{i_{2}} b_{i_{2}}+\cdots+\alpha_{i_{m}} b_{i_{m}}, \quad(m \leq n)
$$

Since $d(\mathcal{A}) \subseteq \mathcal{J}_{k}$ for every $k \in\{1,2, \ldots, n\}$, we may assume that $d(\mathcal{A}) \subseteq \mathcal{J}_{i_{1}}=\mathcal{B}-\left\{b_{i_{1}}\right\}$. Therefore, we have

$$
d\left(a_{0}\right)=\alpha_{i_{1}} b_{i_{1}}+\alpha_{i_{2}} b_{i_{2}}+\cdots+\alpha_{i_{m}} b_{i_{m}} \in \mathcal{J}_{i_{1}} .
$$

The previous equation shows that $b_{i_{1}} \in \mathcal{J}_{i_{1}}$, which is a contradiction. This contradiction proves our assertion that $d$ is identically zero on $\mathcal{A}$.

Acknowledgements The author is greatly indebted to the referee for his/her valuable suggestions. Moreover, I would like to express my deep appreciation to Professor Ajda Fošner for her help to improve this article.

Open Access This article is distributed under the terms of the Creative Commons Attribution 4.0 International License (http:// creativecommons.org/licenses/by/4.0/), which permits unrestricted use, distribution, and reproduction in any medium, provided you give appropriate credit to the original author(s) and the source, provide a link to the Creative Commons license, and indicate if changes were made. 


\section{References}

1. Ashraf, M.; Rehman, N.: On commutativity of rings with derivations. Result. Math. 42, 3-8 (2002)

2. Ashraf, M.; Rehman, N.; Ali, S.; Mozumder, M.R.: On generalized $(\sigma, \tau)$-biderivations in rings. Asian Eur. J. Math. 4, 389-402 (2011)

3. Brešar, M.: Characterizations of derivations on some normed algebras with involution. J. Algebra 152, 454-462 (1992)

4. Ceven, Y.; Ali Öztürk, M.: Some properties of symmetric bi- $(\sigma, \tau)$-derivations in near-rings. Commun. Korean Math. Soc. 22, 487-491 (2007)

5. Dales, H.G.: Banach Algebras and Automatic Continuity, London Math. Soc. Monographs, New Series, vol. 24. Oxford University Press, New York (2000)

6. Divinsky, N.: On commuting automorphisms of rings. Trans. R. Soc. Can. Sect. III(49), 19-22 (1955)

7. Eshaghi Gordji, M.; Gharetapeh, S.K.; Savadkouhi, M.B.; Aghaei, M.; Karimi, T.: On cubic derivations. Int. J. Math. Anal. 51, 2501-2514 (2010)

8. Eshaghi Gordji, M.; Bavand Savadkouhi, M.: Stability of cubic and quartic functional equations in non-Archimedean spaces. Acta Appl. Math. 110, 1321-1329 (2010)

9. Hosseini, A.; Hassani, M.; Niknam, A.: On the range of a derivation. Iran. J. Sci. Technol. Trans. A Sci. 38, 111-115 (2014)

10. Hosseini, A.; Hassani, M.: Some achievements on two variable $\sigma$-derivations. J. Math. Ext. 8, $93-108$ (2014)

11. Hassani, M.; Hosseini, A.: On two variable derivations and generalized centralizers. J. Adv. Res. Pure Math. 6, 38-51 (2014)

12. Hayati, B.; Eshaghi Gordji, M.; Bavand Savadkouhi, M.: Stability of ternary cubic derivations on ternary frchet algebras. Aust. J. Basic Appl. Sci. 5, 1224-1235 (2011)

13. Jung, Y.S.: Results on the range of derivations. Bull. Korean Math. Soc. 37, 265-272 (2000)

14. Jung, Y.S.: On left derivations and derivations of Banach algebras. Bull. Korean Math. Soc. 35, 659-667 (1998)

15. Jung, Y.S.; Park, K.H.: Noncommutative versions of the Singer-Wermer conjecture with linear left $\theta$-derivations. Acta Math. Sin. English Ser. 24, 1891-1900 (2008)

16. Kaplansky, I.: Projections in Banach algebras. Ann. Math. 53(2), 235-249 (1951)

17. Mathieu, M.: Where to find the image of a derivation. Banach Center Publ. 30, 237-249 (1994)

18. Oukhtite, L.; Mamounib, A.; Beddani, C.: Derivations and Jordan ideals in prime rings. J. Taibah Univ. Sci. 8, 364-369 (2014)

19. Ali Öztürk, M.; Yazarli, H.: Some results on symmetric bi- $(\sigma, \tau)$-derivations in near-rings. Miskolc. Math. Notes 11, 169-173 (2010)

20. Posner, E.C.: Derivations in prime rings. Proc. Am. Math. Soc. 8, 1093-1100 (1957)

21. Pinter-Lucke, J.: Commutativity conditions for rings: 1950-2005. Expo. Math. 25, 165-174 (2007)

22. Rehman, N.: On commutativity of rings with generalized derivations. Math. J. Okayama Univ. 44, 43-49 (2002)

23. Saitô, K.; Maitland Wright, J.D.: On defining AW*-algebras and rickart C*-algebras (2015). arXiv:1501.02434v1 [math. $\mathrm{OA}]$

24. Singer, I.M.; Wermer, J.: Derivations on commutative normed algebras. Math. Ann. 129, 260-264 (1955)

25. Thomas, M.P.: The image of a derivation is contained in the radical. Ann. Math. 128, 435-460 (1988)

26. Yang, S.Y.; Bodaghi, A.; Mohd Atan, K.A.: Approximate cubic $*$-derivations on Banach $*$-algebras. Abstr. Appl. Anal. 1, $877-884(2012)$ 